Academic Essay in Astronomy

\title{
How Does an Aurora Occur?
}

\author{
Jeffrey $\mathrm{Wu}^{1}$
}

${ }^{1}$ Veterans Elementary school, Ellicott City, MD 21043, USA

*Correspondence: tiedanjeffrey@gmail.com

(Received: 12/31/2020; Accepted: 01/24/2021; Published: 06/11/2021)

DOI: https://doi.org/10.37906/isteamc.2021.8

\begin{abstract}
People have been amazed about aurora since when they discovered it, and have also observed many shapes that the aurora comes in such as glow, homogeneous arc, rayed arc, rising vapor column, corona to name a few. This paper examines how does an aurora occur, as well as where and when to see it.
\end{abstract}

Keywords: Auroras, Aurora shapes, History of auroras, Aurora Borealis

\section{Introduction}

The night sky is a beautiful place with stars, comets, and the moon. But there is something you are forgetting. It would never be as beautiful if auroras didn't exist. Prehistoric humans have been fascinated about auroras and even drew pictures of auroras in caves. The earliest picture of an aurora was called "macaronis" and was drawn about 30,000 years ago (NASA-The History of Auroras, n.d.). Aurora was not talked about until 3,600 B.C. Many people have beliefs about aurora and have made folklore about them. Hunters that live in cold places think that an aurora shows the spirit of their ancestors and helps them hunt. In modern age, Maida Withers created a new dance called "Dance of the Aurora" inspired by an aurora in 2001. The dance is about the trip from the sun to the earth to produce an aurora. Auroras have impacted millions of humans and has changed thousands of lives.

Aurora mysteries have questioned humanity for thousands of years with the questions: "How does it happen?", "Why does it look that way?", and "Why does it have the colors that it has?"

\section{How does it happen?}

If you see an aurora close to the North Pole, then it is called Aurora Borealis. But if you see an aurora close to the South Pole, then it is called Aurora Australis. Aurora Borealis was named by Galileo Galilei. Aurora Borealis was named after the roman god of dawn, Aurora, and the Greek wind of the north, Boreas (User, 2015b). He named it that way because he thought it has something to do with light (NASA-The History of Auroras, n.d.). Aurora Australis, however was named by James Cook. James Cook named it that way because Australis is the Latin word for "southern" (10 awesome aurora facts - Social Media Blog Bureau of Meteorology, n.d.).

Kristen Birkeland was the first person to find out the cause of auroras in the 1900's. He found that solar flares coming from the sun can excite atoms in the atmosphere and cause them to give light (Figure 1). Solar flare is made of protons, electrons, and alpha particles. (What are the Northern Lights (Aurora 
Borealis)?, n.d.). Excitation means that, when solar flare crashes into an atom in the atmosphere, the solar flare transfers the energy to the atom. Then the electrons in the atom go further away from the nucleus (core) of the atom. When the atom cools down and the electrons come back to their normal place, it then produces light (10 Bright Facts About the Northern Lights, n.d.).

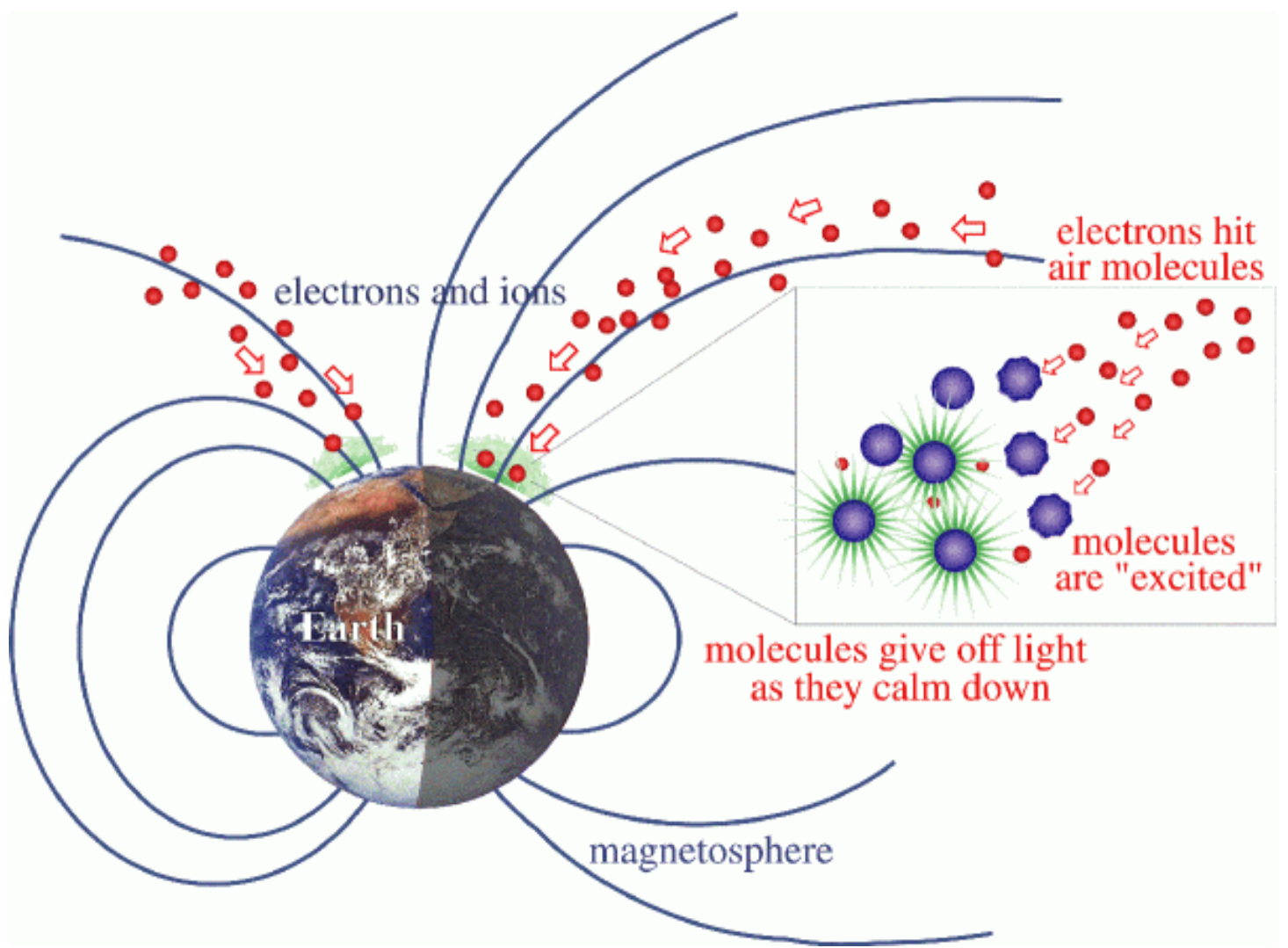

Figure 1. The electrons get pulled by the magnetosphere and the electrons hit atoms in the atmosphere. Then the atoms excite light. (Imster, 2017)

Solar wind is what carries the solar flare to earth. The magnetic field has invisible lines of force that pull the solar flare to the poles creating an aurora as shown in Figure 1. Sometimes the solar wind gets bounced off of the lines of force which are why solar winds need to pass one million miles per hour to enter earth's atmosphere.

\section{Where and when to see an aurora}

Most auroras happen in The Aurora Zone. The Aurora Zone passes though Greenland, Siberia, Scandinavian, and Alaska. You can also see an aurora near the South Pole, but it is harder to see because there are no close counties near the South Pole. You also wouldn't want to go to Antarctica to view aurora, so you would go north to view an aurora. (Aurora Forecast Geophysical Institute, n.d.).

To view Aurora clearly, you should watch it in the darkest night possible. Furthermore, a night with a new moon is better than a night with a full moon. The best months to see an aurora are October, November, and April. Some years are better to view Aurora than other years because of the sun's 11-year sunspot cycle. An aurora substorm happens when solar wind is intense and makes the Aurora Zone extend closer to the equator. Therefore, it will be a great year to view Aurora every 11 years. 2024 and 
2025 will be great years to view the Aurora Borealis (Northern Lights or Aurora Borealis Explained, n.d.). The International Space Station stays around the same height where an aurora is created so you can see a side view of an aurora (O'Neill, 2019). (Figure 2)

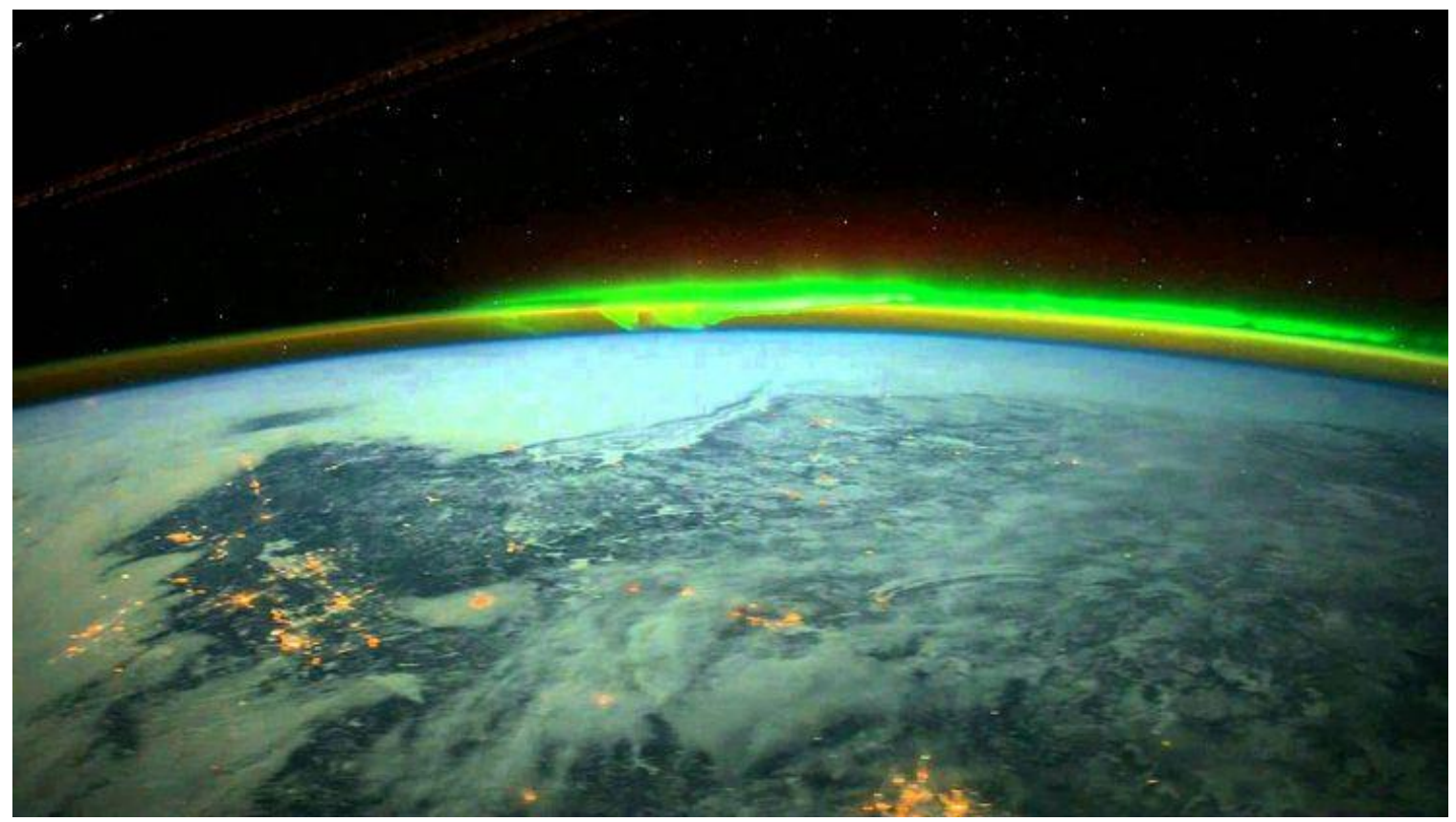

Figure 2. This is the side view of an aurora (O'Neill, R., 2019)

If you happen to see an aurora, return to the same spot 27 days after, there's a good chance that you will see another one. An aurora usually lasts for 15-30 minutes but if you're really lucky, then you might be able to see it for the whole night. That seems very short, but remember that an aurora only happens if solar flare from the sun crashes into the particles in the atmosphere. So as the earth rotates, the solar flare might move into a different space or the magnetic field will deflect the solar flare to stop the aurora.

\section{What can you observe?}

Auroras can take different shapes, which are homogeneous arc, rayed arc, striated band, corona, rising vapor column, pulsating, veil, and active aurora. The glow is just a glow above the horizon as shown in Figure 3. The homogeneous arc is basically an arc. The rayed arc consists of multiple rayed lines. The striated band is a thick line made up of multiple small lines. The corona has rays shooting out from one point. The rising vapor column is like the window curtain in your house. The pulsating aurora happens after intense aurora activity. The veil aurora also happens after intense aurora activity. The active aurora is an aurora that moves rapidly in the sky.

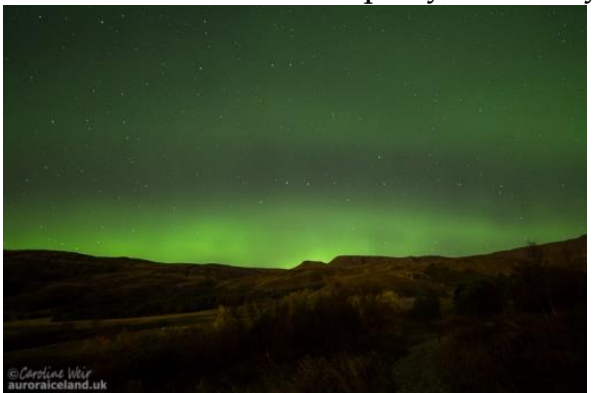

Glow

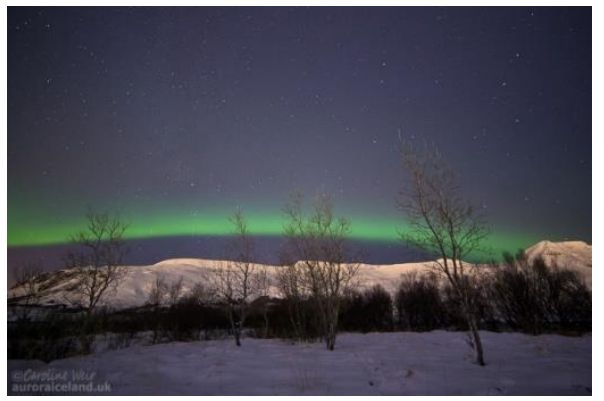

Homogeneous Arc 


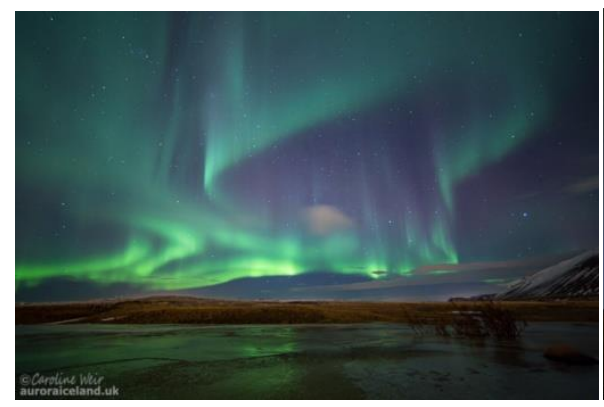

Rayed Arc

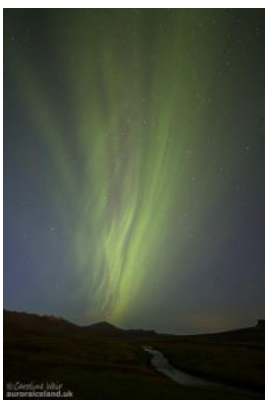

Striated Band

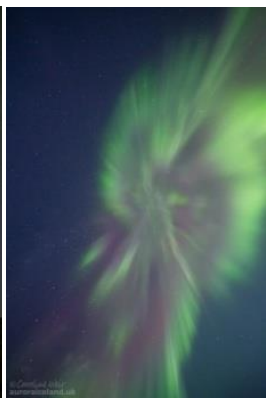

Corona Rising

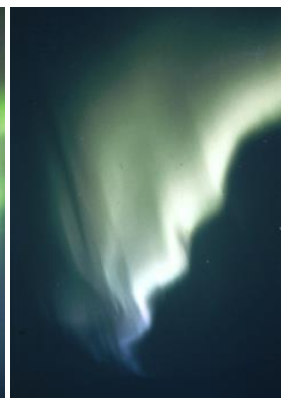

Vapor Column

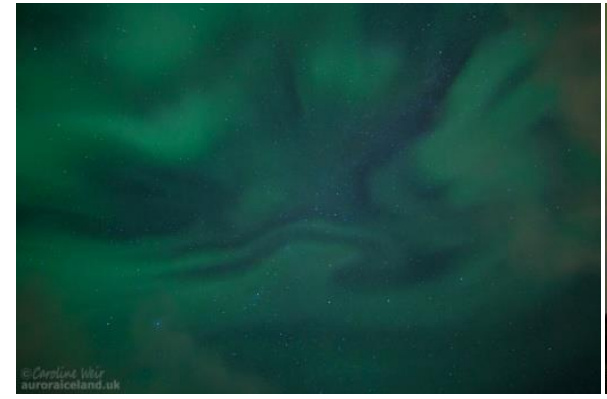

Pulsating

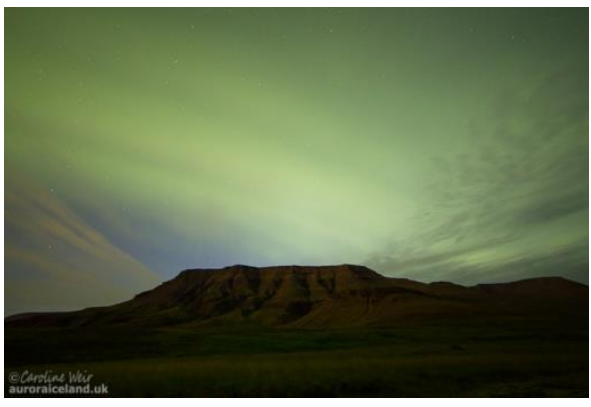

Veil

Figure 3. These are the shapes that aurora can make (Examples of different forms, structures and colors of the aurora borealis. (n.d.).)

An aurora can have different colors such as red, yellow, green, blue, violet (purple), and pink. Usually, solar flares collide into the atoms in the atmosphere at 60-150 miles above earth surface and this produces a green aurora. Also, our eyes can detect color green better than any other colors. Hence, making green is the most common color for a viewable aurora. White, yellow and pink are the rarest colors because red, green, and blue have to mix together in a way get white, yellow, or pink. When a solar flare crashes into nitrogen atoms below 60 miles above the ground, then it produces a bluish-purple light. When a solar flare crashes with an oxygen atom more than 60 miles above the ground, then it produces a greenish yellow light. Occasionally, solar flare can crash into an oxygen atom above 200 miles and produce a red light (User, 2015). An aurora has ultraviolet light which is dangerous to humans' eyes but there is so little of it that humans are not harmed by it.

Sometimes, when the aurora is produced, you can hear a sound that is similar to the static of a radio, crackling, or hissing. The noise comes from charged particles that are trapped in a layer of the atmosphere. This usually happens on very cold nights. Earth's geomagnetic disturbances cause the charged particles to discharge with sparks to produce sound (Metcalfe, 2016).

Auroras can also happen on Mars, Jupiter, and Saturn (What Is an Aurora? I NASA Space Place NASA Science for Kids, n.d.)! (Figure 4.) The auroras seen on Mars and Saturn are formed the same way as Earth's Auroras but Jupiter's Aurora complex. Jupiter has a volcanic moon, Io, which sends plasma to Jupiter, though a fraction of Jupiter's auroras are formed by solar flares. If a planet has solar flares and has an atmosphere, then it is likely that the planet will create an aurora. 


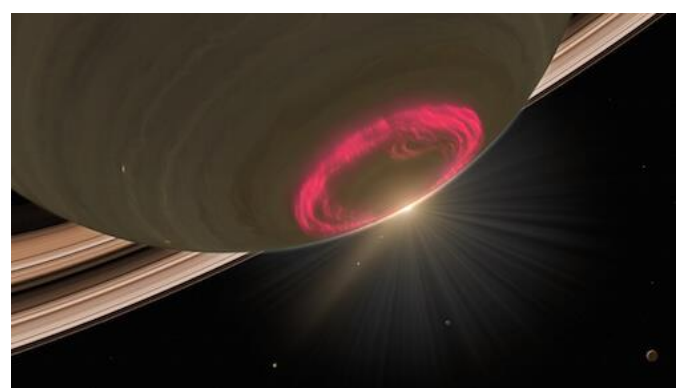

An Aurora on Jupiter

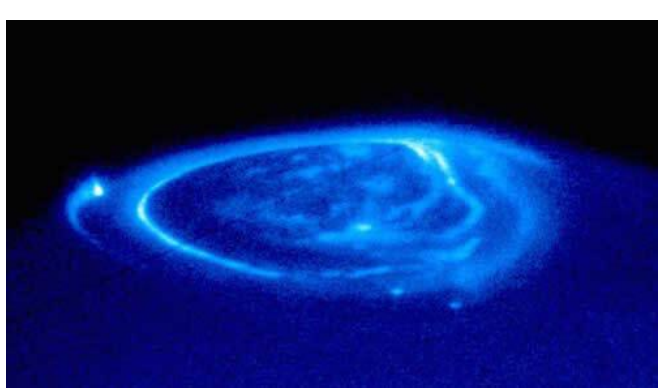

An Aurora on Saturn using ultraviolet light to see it

Figure 4. Auroras happen on Mars and Saturn

\section{Conclusion}

Auroras were a mysterious phenomenon before we figured out what caused it. The cause has fascinated humans to learn more and more about Aurora. The beliefs and history of Auroras back then are still passed on. Auroras will stay with us forever.

\section{References}

Magical Places to View Auroras. (2017, February 16). Travel. https://www.nationalgeographic.com/travel/top-10/7-aurora-destinations/\#close

10 Awesome Aurora Facts (n.d.). Social Media Blog - Bureau of Meteorology http://media.bom.gov.au/social/blog/1114/10-awesome-aurorafacts/eographic.com/travel/top-10/7-aurora-destinations/\#close

Aurora Forecast I Geophysical Institute. (n.d.). Https://Www.Gi.Alaska.Edu/Monitors/Aurora-Forecast. Retrieved December 7, 2020, from https://www.gi.alaska.edu/monitors/aurora-forecast

Imster, E. (2017, February 15). What causes the aurora borealis? EarthSky. https://earthsky.org/earth/what-causes-the-aurora-borealis-or-northern-lights

Metcalfe, T. (2016, October 12). How One Scientist Decoded the Mysterious Sounds of the Northern Lights. Livescience.Com. https://www.livescience.com/56457-scientist-decodedmysterious-northern-lights-sounds.html

NASA - The History of Auroras. (n.d.). NASA. https://www.nasa.gov/mission_pages/themis/auroras/aurora_history.html

Northern Lights or Aurora Borealis Explained. (n.d.). Https://www.northernlightscentre.ca/northernlights.html

O'Neill, R. (2019, September 4). 10 Things You Didn't Know About The Aurora Borealis. TheTravel. https://www.thetravel.com/things-didnt-know-aurora-borealis 
The Northern Lights colors (2015, April 20). The Aurora Zone Https://Www.Theaurorazone.Com/aboutthe-Aurora/the-Science-of-the-Northern-Lights/the-Northern-Lights-Colours.

https://www.theaurorazone.com/about-the-aurora/the-science-of-the-northern-lights/thenorthern-lights-colours 